\title{
Sri Jayewardenepura Breastfeeding Study: Maternal and infant outcomes in relation to the duration of breast feeding
}

\author{
Pubudu de Silva ${ }^{1}$, Sampath Kusumsiri ${ }^{1}$, Ruchira Dissanayake ${ }^{1}$, Rani Wasala ${ }^{2}$ \\ Sri Lanka Journal of Obstetrics and Gynaecology 2009; 31: 88-96
}

\begin{abstract}
Introduction: The WHO emphasizes exclusive breastfeeding (EBF) for six months. In Sri Lanka the EBF for 4-6 months is recommended. There is paucity of data in issues concerning policymaking with regard to defining the optimal duration of EBF in Sri Lanka.
\end{abstract}

Objectives: To compare maternal and infant outcomes in 3 groups of paired mother-infants: four month of EBF, between four months and six months of EBF and six months of EBF.

Methods: A randomized interventional study. Only normal mother-infant pairs were included. All groups educated on weaning and evaluated monthly. Assessments of anthropometry, morbidity, motor development, lactational amenorrhea, micronutrition status (Haemoblobin level and serum iron) were done in all three groups.

Results: Less number of infants with growth faltering $(p=0.093)$ and acute gastroenteritis $(p=0.135)$ while more infants crawled, longer period of lactational amenorrhea $(p=0.276)$ and higher mean maternal weight loss $(p=0.152)$ observed in 6 months EBF group compared to the other two groups. No significant differences in mean serum iron concentration and mean haemoglobin concentration among the three groups $(p=0.928$ and $\mathrm{p}=0.921$ respectively).

There were no significant differences in the above factors among the three groups at one year of age.

Conclusions: Six months EBF has favorable out comes in terms of weight faltering, acute gastroenteritis, motor development, lactational amenorrhea and maternal weight loss. EBF for

\footnotetext{
${ }^{1}$ Medical Officer,

${ }^{2}$ Paediatrician, Sri Jayewardenepura General Hospital, Sri Jayewardenepura Kotte, Sri Lanka.

Corresponding author: Pubudu de Silva

E-mail: pubududesilva@ymail.com
}

six months has not effected the serum iron concentration and heamoglobin level in this study sample. There were no significant benefits at one year of age.

Key words: Six months exclusive breast feeding, maternal lactational amenorrhea, infant micronutrition status.

\section{Introduction}

The benefits of breast-feeding for both child and mother in terms of nutrition, immunological protection, anti-infective, biochemical, anti-allergic and contraceptive effects, and emotional satisfaction have been widely documented. Over the past decade this has been recognized by public health development organizations, public health experts and governments around the world.

Although breastfeeding continues to be the norm in developing countries the period of exclusive breastfeeding after birth is often short ${ }^{1}$. Benefits of exclusive breastfeeding led to a recommendation by the World Health Organization (WHO) that it should be done for the first six months of life ${ }^{2}$, consistent with a previous recommendation by UNICEF (United Nations Children's Education Fund).

Report of the expert consultation on the optimal duration of exclusive breastfeeding (EBF) revealed that exclusive breastfeeding up to 6 months confers several benefits on the infant and the mother. However, exclusive breastfeeding to 6 months can lead to iron deficiency in susceptible infants ${ }^{3,4}$. In addition, the available data are insufficient to exclude several other potential risks with exclusive breastfeeding for 6 months, including growth faltering and other micronutrient deficiencies, in some infants ${ }^{1,3}$. Also it should be noted that the review was based on two small controlled trials and 17 observational studies that varied in both quality and geographic provenance.

The paucity of data from the low income Asian communities has delayed Sri Lanka from adapting WHO recommendation ${ }^{6}$. This study was carried out as a preliminary investigative study to detect the 
feasibility and safety in carrying out a population based study in a wider community setting which in turn will render adequate information for revision of breastfeeding policies.

\section{Objectives}

\section{Primary objective}

To compare maternal and infant outcomes in 3 groups of paired mother-infants: four month of exclusive breastfeeding (Group 1), more than four months to less than six months of exclusive breastfeeding (Group 2) and six months of exclusive breastfeeding (Group 3).

\section{Secondary objective:}

\section{Infant outcomes investigated:}

- The incidence of growth faltering, acute gastroenteritis, respiratory tract infections and bronchiolitis (or bronchial asthma)

- The distribution of Haemoglobin $(\mathrm{Hb})$ level and serum iron levels

- The description of gross motor development

\section{Maternal outcomes investigated:}

- Maternal weight loss

- Duration of maternal lactational amenorrhea

- Compliance in mothers who were advised for six months EBF.

- Reasons for starting weaning before six months in mothers who were advised to breastfeed exclusively for six months.

\section{Materials and methods}

Study setting: Study was carried out at Sri Jayewardenepura General Hospital (SJGH), which is a 1001 bedded tertiary care hospital teaching hospital situated in the capital city of the country. In 2004 there were 56,803 admissions with 4846 deliveries. The subjects were recruited from the two maternity wards, Ward 02 and Ward 09. It mainly caters for the middle lower class and above.

Study population: Mothers who delivered in SJGH hospital with their respective infant.

Enrollment: Only after obtaining informed written maternal consent for the study. It was obtained by a medical officer before leaving the hospital, after childbirth. Ethical clearance to the study was given by the Ethical Review Committee of Faculty of Medicine, University of Colombo.
Study period: Enrollment of babies for the study was done from 22nd of September 2003 to 10th of November 2003. Follow-up of the selected babies was carried out from 22nd of October 2003 to 10th of November 2004.

Study design: The study was in 2 parts:

1. A prospective observational study from birth to 4 months

2. Followed by a randomized intervention trial to determine the impact of complementary foods from 4 to 6 months and a follow-up period from 6 to 12 months.

\section{Inclusion criteria:}

Mothers who are:

- Unemployed or not employed outside the home before 6 months postpartum,

- 18 years or older

- Healthy mothers (not taking medication on a regular basis)

- Willing to come to the SJGH for monthly followups

- With a Hb level of $11 \mathrm{mg} / \mathrm{dl}$ or more

Infants who are:

- Term babies (from 37 to 42 weeks of period of amenorrhea)

- Normal and healthy

- Birth weight more than $2,500 \mathrm{~kg}$

\section{Exclusion criteria:}

Mothers:

- Diagnosed of anaemia during pregnancy

- Who are employed during pregnancy

- Who were already breastfeeding other older siblings or who were already caring for another infant at the time of the current delivery

Infants:

- Admitted to the Neonatal Intensive Care Unit (NICU) after delivery

- Low birth weight infants (birth weight less than $2,500 \mathrm{~kg}$ )

- Neonates with congenital anomalies

- With severe medical conditions that might interfere with feeding or growth

- Who suffered from illnesses other than acute gastroenteritis, upper respiratory tract infection, bronchioloitis, bronchial asthma or atopic diseases; during the study period. 
- Neonates of mothers' with contraindication for breastfeeding

- Twins

\section{Study methodology:}

During the recruiting period 300 mothers of consecutive deliveries who fulfilled the criteria were enrolled. At the time of recruitment mothers were given information on the correct procedure of breast feeding and the benefits of breastfeeding on a one to one basis by a paediatric medical officer and the nursing sister of the paediatric ward. At the time of enrolment the socio-demographic data and pregnancy information were collected.

Follow-up of the infants and mothers were done at three months, four months, five months, six months, seven months, nine months and twelve months of age. Mothers were given appointment days to visit the paediatric ward of SJGH for follow-up visits. They were informed by letters and telephone calls one week prior to their appointment dates by a paediatric medical officer. The data from defaulters were not included. Anthropometry measurements (detailed in data collection), assessment of morbidity (detailed in data collection), assessment of motor development (detailed in data collection) and assessment of lactational amenorrhea were done during each visit. $\mathrm{Hb}$ level and serum iron were measured during the visit at six months of age.

During the first visit at three months of age all mothers of babies who fulfilled the criteria for the continuation in the study were educated on weaning on one to one basis by a paediatric medical officer. At the first visit the infants were randomly allocated to two groups. The randomization was done by week of birth to facilitate provision of feeding instructions to each group during their visits to the Paediatric Ward. One group was advised to start weaning at the end four months and the other group was advised to start weaning at the end of six months of age. Infants were examined by the paediatric medical officers and clarifications were sought from the Consultant Paediatrician.

The assessment at six months of age was done by a paediatric medical officer who had not participated in the randomization process to minimize any outcome reporting bias.

Data collection: All data were collected using a pretested standard questionnaire. The collection was done by a trained paediatric medical officer. An interview with the mother, the bed head ticket, the mother's pregnancy card and the child health development record were used to collect the data.

The following data were collected:

\section{Anthropometry}

Weights of infants are measured on a scale accurate to the nearest $100 \mathrm{~g}$. The scale is calibrated weekly by using standard weights. Infant recumbent length is measured to the nearest $1 \mathrm{~cm}$ and head circumference to the nearest $0.5 \mathrm{~cm}$.

Maternal weight is measured to the nearest $100 \mathrm{~g}$ and the scale is calibrated weekly.

Growth faltering is taken as downward deviation of curve for at least 2 plots.

\section{Assessment of morbidity}

Morbidity is assessed as the number of events occurred and the number of days for each event lasted for diarrhea, respiratory tract infection, bronchiolitis or bronchial asthma and atopic diseases.

\section{Assessment of motor development}

At each clinic visit, mothers were asked whether their infants could perform any of the following 10 motor milestones and, if so, when it first occurred:

1) While lying face down, the infant can raise the head and look forward

2) While lying face down, the infant can raise the head and chest, supporting the body with the arms;

3) The infant can regularly roll over (from back to front)

4) The infant can crawl (sustained movement)

5) From a lying down position, the infant can get into a sitting position

6) The infant can stand while holding on to furniture

7) The infant can pull to a standing position

8) The infant can walk while holding on to furniture ("cruising")

9) The infant can stand alone (for $30 \mathrm{~s}$ )

10) The infant can walk unaided

\section{Assessment of lactational amenorrhea}

At each clinic visit, mothers were asked if they experienced any menstrual bleeding since the previous visit, and if so, the dates and duration of each episode

Sri Lanka Journal of Obstetrics and Gynaecology 
were recorded. Information on the use of hormonal contraceptives was also collected. The definition of the first menstrual period was based on the following criteria: a) it lasted $>1$ day, b) it occurred after $56 \mathrm{~d}$ postpartum and c) it was followed by an interval of at least $21 \mathrm{~d}$ but not $>70 \mathrm{~d}$ before the next bleed, as previously described.

\section{Micronutrition status}

Haemoblobin level and serum iron (colorimetric method) are measured at 6 months of age.

\section{Statistical analysis}

Data were analysed using SPSS 11. For comparison of discrete variables the chi square was used.
For comparison of continuous variables ANOVA was used.

\section{Results}

At three months 147 out of 300 came for the first follow-up. There was no significant difference in socio demographic, parity, pregnancy and infant morbidity between the 147 who turned up for follow-up and 153 who failed to turn up. At six months assessment this was reduced to 98 and at one year assessment to 79 . The compliance rate for the follow-ups at 6 months was $32.6 \%$ and at 1 year $26.3 \%$. In the 6 months EBF some mothers have started weaning after 4 months but before completion of 6 months. The resulted sample sizes at 6 months and at 1 year are described in Table 1.

Table 1. Final number of mother-infants pairs eligible for analysis

\begin{tabular}{lccc}
\hline Follow up & $\mathbf{4}$ months & $\mathbf{> 4}$ months to $<\mathbf{6}$ months & $\mathbf{6}$ months \\
\hline 6 months & 44 & 30 & 21 \\
1 year & 32 & 16 & 17 \\
\hline
\end{tabular}

Maternal characteristics of the three groups are described in Table 2.

Table 2. Maternal characteristics of the three groups

\begin{tabular}{lccc}
\hline Follow up & $\mathbf{4}$ months & $>$ 4 months to $<6$ months & $\mathbf{6}$ months \\
\hline O/L or above & $42(95.5 \%)$ & $28(93.3 \%)$ & $20(100 \%)$ \\
Primi para & $20(45.5 \%)$ & $15(50.0 \%)$ & $08(38.1 \%)$ \\
Multi para & $24(54.5 \%)$ & $15(50.0 \%)$ & $13(61.9 \%)$ \\
Mean monthly income (Rs.) & 11102.27 & 14916.67 & 10500.00 \\
\hline
\end{tabular}

There were no significant differences regarding the maternal education level $(p=0.515)$, parity $(p=0.702)$ and the mean monthly income $(\mathrm{p}=0.059)$ of the household among the three groups.

In the six months EBF group $40.38 \%$ of mothers were compliant to EBF for six months. On inquiry the main reasons for abandoning six months of EBF by the mothers are due to the advice given by the health care workers $(56.25 \%)$. The other main reason was the mothers' perception of reduced secretion of breast milk (31.25\%).

\section{Evaluation at 6 months}

At six months of age the least incidence of weight faltering, acute gastroenteritis (AGE) and respiratory tract infections were observed in the 6 months EBF group. The highest mean haemoglobin level and serum iron level were also seen in the 6 months EBF group (Table 3). There was no reporting of bronchiolitis / bronchial asthma or atopic diseases. 
Table 3. Assessment at 6 months

\begin{tabular}{lccccc}
\hline $\begin{array}{l}\text { EBF period } \\
\text { (in months) }\end{array}$ & $\begin{array}{c}\text { Weight } \\
\text { faltering }\end{array}$ & $\begin{array}{c}\text { AGE } \\
\text { incidence }\end{array}$ & $\begin{array}{c}\text { RTI } \\
\text { incidence }\end{array}$ & $\begin{array}{c}\text { Mean Hb } \\
(\mathbf{m g} / \mathbf{d l})\end{array}$ & $\begin{array}{c}\text { Mean S. iron } \\
(\mu \mathrm{mol} / \mathbf{l})\end{array}$ \\
\hline & $12(27.3 \%)$ & $08(18.6 \%)$ & $17(39.5 \%)$ & 11.37 & 8.55 \\
4 & $05(17.2 \%)$ & $08(27.6 \%)$ & $11(37.9 \%)$ & 11.38 & 8.00 \\
6 & $01(04.8 \%)$ & $01(05.0 \%)$ & $07(35.0 \%)$ & 11.44 & 8.80 \\
Significance & $\mathrm{p}=0.093$ & $\mathrm{p}=0.135$ & $\mathrm{p}=0.942$ & $\mathrm{p}=0.921$ & $\mathrm{p}=0.928$ \\
\hline
\end{tabular}

There was no significant relationship between infant weight faltering with AGE $(p=0.994)$ or RTI $(\mathrm{p}=0.845)$ (Table 4 and Table 5).

Table 4. Comparison of infant weight faltering with incidence of AGE at six months

\begin{tabular}{|l|c|c|}
\hline \multirow{2}{*}{ Infant weight faltering at $\mathbf{6}$ months } & \multicolumn{2}{|c|}{ AGE incidence } \\
\cline { 2 - 3 } & Present & Absent \\
\hline Present & $03(17.6 \%)$ & $14(82.4 \%)$ \\
\hline Absent & $13(17.6 \%)$ & $61(82.4 \%)$ \\
\hline
\end{tabular}

Table 5. Comparison of infant weight faltering with incidence of RTI at six months

\begin{tabular}{|l|c|c|}
\hline Infant weight faltering at $\mathbf{6}$ months & \multicolumn{2}{|c|}{ RTI incidence } \\
\hline & Present & Absent \\
\cline { 2 - 3 } Present & $06(35.3 \%)$ & $11(64.7 \%)$ \\
\hline Absent & $28(37.8 \%)$ & $46(62.2 \%)$ \\
\hline
\end{tabular}

The level of motor development was more in 6 months EBF group (Table 6).

Table 6. Level of Motor Development at six months

\begin{tabular}{|c|c|c|c|}
\hline \multirow{2}{*}{$\begin{array}{c}\text { EBF period } \\
\text { (in months) }\end{array}$} & Range & Median & Mode \\
\cline { 2 - 4 } & $2-5$ & 03 & 03 \\
\hline 4 & $3-5$ & 03 & 03 \\
\hline $4-6$ & $3-6$ & 04 & 04 \\
\hline 6 & & & \\
\hline
\end{tabular}


The period of lactational amenorrhea (months) was more $(p=0.276)$ in 6 months EBF group (Table 7).

Table 7. Period of lactational amenorrhea (months) at six months

\begin{tabular}{|l|c|c|c|c|}
\hline \multirow{2}{*}{$\begin{array}{l}\text { EBF period } \\
\text { (in months) }\end{array}$} & \multicolumn{3}{|c|}{ Period of lactational amenorrhea (months) } \\
\cline { 2 - 5 } & Range & Mean & Median & Mode \\
\hline 4 & $2-6$ & 4.017 & 4.00 & 06 \\
\hline $4-6$ & $2-7$ & 4.781 & 5.25 & 06 \\
\hline
\end{tabular}

The most maternal weight loss $(\mathrm{kg})$ was seen in the 6 months EBF group ( $\mathrm{p}=0.152)$ (Table 8).

Table 8. Maternal weight loss at six months

\begin{tabular}{|l|l|l|c|}
\hline \multirow{2}{*}{$\begin{array}{l}\text { EBF period } \\
\text { (in months) }\end{array}$} & Maternal weight loss (kg) & Median \\
\cline { 2 - 4 } & Range & $-0.63(-1.33 \%)$ & -5.0 \\
\hline 4 & $-1.5-+0.26$ & $+0.31(+3.7 \%)$ & 0.0 \\
\hline $4-6$ & $-0.88-+1.5$ & $+0.47(+8.5 \%)$ & 0.0 \\
\hline
\end{tabular}

\section{Evaluation at 1 year}

At one year of age the least incidence of weight faltering and acute gastroenteritis (AGE) and atopic diseases were observed in the between 4 and 6 months EBF group. The least incidence of respiratory tract infections and bronchial asthma were observed in the 4 months EBF group (Table 9).

Table 9. Assessment at 1 year of age

\begin{tabular}{|l|l|l|l|l|l|}
\hline $\begin{array}{l}\text { EBF period } \\
\text { (in months) }\end{array}$ & $\begin{array}{l}\text { Weight } \\
\text { faltering }\end{array}$ & $\begin{array}{l}\text { AGE } \\
\text { incidence }\end{array}$ & $\begin{array}{l}\text { RTI } \\
\text { incidence }\end{array}$ & $\begin{array}{l}\text { Bronchial asthma } \\
\text { incidence }\end{array}$ & $\begin{array}{l}\text { Atopic } \\
\text { diseases } \\
\text { incidence }\end{array}$ \\
\hline 4 & $08(24.2 \%)$ & $10(30.3 \%)$ & $21(63.6 \%)$ & $02(06.1 \%)$ & $02(6.1 \%)$ \\
$4-6$ & $01(06.3 \%)$ & $02(12.5 \%)$ & $11(68.8 \%)$ & $01(06.3 \%)$ & $00(0.0 \%)$ \\
6 & $07(41.2 \%)$ & $06(35.3 \%)$ & $14(82.4 \%)$ & $02(17.6 \%)$ & $01(5.9 \%)$ \\
Significance & $\mathrm{p}=0.065$ & $\mathrm{p}=0.292$ & $\mathrm{p}=0.210$ & $\mathrm{p}=0.561$ & $\mathrm{p}=0.395$ \\
\hline
\end{tabular}


There was no significant relationship $(p=0.127)$ between infant weight faltering with AGE (Table 10).

Table 10. Comparison of infant weight faltering with incidence of AGE at one year

\begin{tabular}{|l|c|c|}
\hline Infant Weight Faltering at 1 year & \multicolumn{2}{|l|}{ AGE incidence } \\
\hline & Present & Absent \\
\hline Present & $02(12.5 \%)$ & $14(87.5 \%)$ \\
\hline Absent & $16(32.0 \%)$ & $34(68.0 \%)$ \\
\hline
\end{tabular}

There was significant relationship $(\mathrm{p}=0.016)$ between infant weight faltering with RTI (Table 11).

Table 11. Comparison of infant weight faltering with incidence of RTI at one year

\begin{tabular}{|l|l|l|}
\hline Infant Weight Faltering at 1 year & $\begin{array}{l}\text { RTI incidence } \\
\text { Present }\end{array}$ & Absent \\
\hline Present & $15(93.8 \%)$ & $01(06.3 \%)$ \\
\hline Absent & $31(62.0 \%)$ & $19(38.0 \%)$ \\
\hline
\end{tabular}

The level of motor development was more in 6 months EBF group (Table12).

Table 12. Level of Motor Development at one year

\begin{tabular}{|c|c|c|c|}
\hline \multirow{2}{*}{$\begin{array}{c}\text { EBF period } \\
\text { (in months) }\end{array}$} & Range & Median & Mode \\
\cline { 2 - 4 } & $6-9$ & 08 & 08 \\
\hline 4 & $8-9$ & 09 & 09 \\
\hline $4-6$ & $7-9$ & 09 & 09 \\
\hline 6 & & & \\
\hline
\end{tabular}

The most maternal weight loss $(\mathrm{kg})$ was seen in the 6 months EBF group ( $\mathrm{p}=0.756)$ (Table 13).

Table 13. Maternal weight loss at one year

\begin{tabular}{|c|c|c|c|}
\hline \multirow{2}{*}{$\begin{array}{c}\text { EBF period } \\
\text { (in months) }\end{array}$} & Range & Median & Mode \\
\cline { 2 - 4 } & $+0.5-+2.54$ & $+1.52(+2.51 \%)$ & +1.50 \\
\hline 4 & $-1.03-+1.62$ & $+0.29(+0.33 \%)$ & +1.57 \\
\hline $4-6$ & $+0.29-+3.3$ & $+1.79(+3.18 \%)$ & +0.75 \\
\hline 6 & & & \\
\hline
\end{tabular}




\section{Discussion}

The reduction of follow up compliance rate in the first visit was $51 \%$. However no statistical significance different noted between those who came for follow-up and those who did not. The main reason for poor response was that more than $80 \%$ of the uncomplicated deliveries of normal babies were followed up in the well baby clinics near to the mothers' residence. Therefore only a minor segment of mothers will bring their babies for the SJGH well baby clinic. This may be one of the reasons for the defaulted follow up visits.

\section{Analysis at six months}

Although at six months of age the least incidence of weight faltering $(p=0.062)$, acute gastroenteritis $(p=0.098)$ and respiratory tract infections $(p=0.942)$ were observed in the 6 months EBF group the difference is not significant. As the sample of infants selected was healthy, the weight faltering in the study sample may also be due to AGE or RTI. The $p$ values for the weight faltering occurring due to AGE or RTI are respectively 0.994 and 0.845 . Therefore it seems unlikely that the weight faltering was due to AGE or RTI. This is consistent with the currently available data which also suggests protective effects against gastrointestinal infection and non-protective effect against respiratory tract infection ${ }^{7}$. Strong protective effect against AGE was observed in Belarus ${ }^{8}$. However the morbidity data from developing countries were limited to the two Honduran trials, which had insufficient statistical power to detect any advantage of EBF to six months, and which used hygienically prepared complementary foods ${ }^{9,10}$.

The probable reason for absence of reporting of bronchiolitis / bronchial asthma or atopic diseases in this sample may be due to the low incidence of these in this selected sample. Some reports indicate that breastfeeding does not protect against atopy ${ }^{11}$.

Regarding mean haemoglobin level and serum iron level there was no significant difference in the three groups. The evidence from one trial in Honduras demonstrates poorer iron status in infants exclusively breastfed for six months than the 4 months group ${ }^{4}$. The finding is likely to apply to populations in which maternal iron status and infant endogenous stores of iron are not optimal. In this study only the healthy infants of mothers with HB $11 \mathrm{mg} / \mathrm{dl}$ or above were selected to exclude those with deficient maternal iron status and infant endogenous stores.

In the six months EBF group more infants crawled, longer period of lactational amenorrhea $(p=0.276)$ and higher mean maternal weight loss $(p=0.152)$ was observed compared to the other two groups. Results of studies done in Pakistan, Philippines and Thailand confirmed that breast-feeding plays a significant role in delaying the recovery of ovulation ${ }^{12}$. The Honduras trial revealed infants in the six months EBF groups crawled sooner, and mothers in the same group had significant weight loss and were more likely to be amennorrheic at 6 months compared to the 4 months EBF group $9,10,13$. Even though the Honduran trials reported more rapid motor development they were reported to be inconsistent and susceptible to observer bias by the expert consultation on the optimal duration of exclusive breastfeeding.

\section{Analysis at one year}

Assessment at one year of age revealed less weight faltering $(p=0.065)$ in the $>4$ to $<6$ months group compared to other two groups. The weight faltering at one year of age was significantly associated with RTI $(p=0.016)$ but not with AGE $(p=0.127)$. More percentage of weight faltering was seen in the six months EBF group probably due to more AGE and RTI observed in this group and different feeding practices.

There were no significant associations regarding acute gastroenteritis $(p=0.292)$, respiratory tract infections $(p=0.210)$, bronchiolitis / bronchial asthma $(p=0.561)$, or atopic diseases $(p=0.395)$ even though high incidence was observed in the six months EBF. It is likely that the high incidences observed are more due to chance as the comparing sample size is critically small and due to the presence of directly influencing multiple factors at one year of age other than initial feeding methods.

In the six months EBF group more infants can stand alone and higher mean maternal weight loss ( $p=0.756$ ) was observed compared to the other two groups.

\section{Conclusion}

Six months EBF for normal healthy infants of unemployed mothers have benefits at six months of age. This group has no unfavourble effects on serum iron and haemoglobin levels at this age. Multi centre field trials should be launched and outcome should be evaluated.

There is no advantage for the six months EBF group when considering the results at one year of age. This is due to the presence of multiple factors that are directly influencing the results other than initial feeding methods. 


\section{References}

1. Black RE, Victoria CG. Editorial: Optimal duration of exclusive breastfeeding in low income countries. BMJ 2002; 325: 1252-53.

2. Fifty-fourth World Health Assembly. Infant and young child nutrition. World Health Assembly 54.2, Agenda item 13.1, 18 May 2001.

3. Lartey A, Manu A, Brown KH, Dewey KG. Predictors of micronutrient status among six to twelve-month-old breastfed Ghanaian infants. Journal of Nutrition 2000; 130 : 199-207.

4. Dewey KG, Cohen RJ, Rivera LL, Brown KH. Effects of age of introduction of complementary foods on iron status of breast-fed infants in Honduras. American Journal of Clinical Nutrition 1998; 67: 878-84.

5. de Alwis Y, Warnasuriya N, Sirisena LAW. Duration of exclusive breastfeeding and its relationship to growth faltering Annual Scientific Congress, October 2003, Colombo, Sri Lanka, Abstract: page 45.

6. World Health Organization. The optimal duration of exclusive breastfeeding. Report of an expert consultation. Geneva, 28-31 March 2001 (WHO/FCH/CAH/01.24).

7. Arifeen S, Black RE, Antelman G, Baqui A, Caulfield L BeckerS. Exclusive breastfeeding reduces acute respiratory infections and diarrhea deaths among infants in Dhaka slums. Pediatrics 2001; 108(4): e67.

8. Betran AP, de Onis M, Lauer JA, Villar J. Ecological study of effect of breastfeeding on infant mortality in Latin America. BMJ 2001; 323: 303.

9. Dewey KG, Cohen RJ, Brown KH, Rivera LL. Effects of exclusive breastfeeding for four versus six months on maternal nutritional status and infant motor development: Results of two randomized trials in Honduras. Journal of Nutrition 2001; 131: 262-7.

10. Dewey KG, Cohen RJ, Brown KH, Rivera LL. Age of introduction of complementary foods and growth of term, low-birth-weight, breast-fed infants: a randomized intervention study in Honduras. American Journal of Clinical Nutrition 1999; 69(4): 679-86.

11. Mayor S. Breastfeeding does not protect against atopy. BMJ 2002; 325: 675 .

12. Kennedy KI, Rivera R, McNeilly AS. Consensus Statement on the Use of Breast-Feeding as a Family Planning Method. Contraception 1989; 39(5): 477-96.

13. Cohen RJ, Brown KH, Canahuati J, Rivera LL, Dewey KG. Effects of age of introduction of complementary foods on infant breast milk intake, total energy intake, and growth: a randomized intervention study in Honduras. Lancet 1994; 343: 288-93. 